\title{
Assessing the Strands of Student Proficiency in Elementary Algebra
}

\author{
William G. McCallum
}

May, 2005

\section{Algebra and Functions}

In order to assess something, you have to have some idea of what that something is. Algebra means many different things in today's schools (for a discussion, see [RAN03], Chapter 4). In particular, the study of algebra is often blended with the study of functions. Although it is true that the notion of a function is lurking behind much of beginning algebra, and that there is algebraic aspect to many of the tasks we want our students to carry out with functions, the conglomeration of algebra and functions has led to considerably muddy waters in the teaching of algebra. Therefore, I'd like to spend some time clarifying my own stance before talking about how to assess proficiency. In the process, while acknowledging other possible uses, I will use the word algebra to mean elementary algebra, that is, the study of algebraic expressions and equations in which the letters stand for numbers.

In the progression of ideas from arithmetic to algebra to functions, there is an increase in abstraction at each step, and the increase at the second step is at least as large as that at the first. In the step from arithmetic to algebra, we learn to represent numbers by letters, and calculations with numbers by algebraic expressions. In the step from algebra to functions we learn of a new sort of object, function, and represent functions themselves by letters. There are two complementary dangers in the teaching of algebra, each of which can cause students to miss the magnitude of this step.

The first danger is that functions and function notation appear to the students to be mostly a matter of using a sort of auxiliary notation, so that if a function $f$ is defined by $f(x)=x^{2}-2 x-3$, say, then $f(x)$ is nothing more than a short-hand notation for the expression $x^{2}-2 x-3$. This results in a confusion between functions and the expressions representing them, which in turn leads to a broad area of confusion around the ideas of equivalent expressions and transforming expressions. For example, we want students to understand that $(x+1)(x-3)$ and $(x-1)^{2}-4$ are equivalent expressions, each of which reveals different aspects of the same function. But without a strong notion of function as an object distinct from the expression defining it, the significance of 
equivalence and transformation is lost in a welter of equals signs following the $f(x)$. Students can't see the forest for the trees.

A complementary danger is that algebra appears as a sort of auxiliary to the study of functions. It is common to use multiple representations of functions in order to make concrete the notion of a function as an object in its own right. Functions are represented by graphs, tables, or verbal descriptions, in addition to algebraic expressions. By looking at the same object from different points of view, one aims to foster the notion of its independent existence. Under this approach, algebra provides one way of looking at functions, but functions are not regarded as purely algebraic objects. This solves the problem of students not seeing the forest for the trees, but risks them not being able to see the trees for the forest. Overemphasizing functions when teaching algebra can obscure algebraic structure, and too much attention to graphical and numerical approaches can subvert the central goal of thinking about symbolic representation.

In answering the question posed in the title, I limit myself to the narrower meaning of algebra that I have been using, and interpret proficiency in algebra as largely a matter of proficiency with symbolic representations. I understand proficiency to include the five strands of mathematical competence described in [KSF01]: conceptual understanding, procedural fluency, strategic competence, adaptive reasoning, and productive disposition.

\section{Proficiency in Algebra}

Consider the following common algebraic error, the like of which everybody who teaches calculus has seen at one time or another.

$$
\begin{aligned}
\int \frac{1}{2 x^{2}+4 x+4} d x & =\int \frac{1}{x^{2}+2 x+2} d x \\
& =\int \frac{1}{(x+1)^{2}+1} d x \\
& =\arctan (x+1)+C
\end{aligned}
$$

The student has factored a 2 from the denominator, and then lost it. Students often regard such errors as minor slips, like a wobble while riding a bicycle, and instructors often indulge this point of view with partial credit. The suggested remediation for students who make this sort of error often is lots of drill; lots of practice riding the bicycle. The error is regarded as a matter of procedural fluency; either the student does not know the rules of algebra, or is insufficiently practiced in their execution.

Of course, without further information, it is impossible to diagnose the error precisely. However, it is worth drawing on experience to consider possible diagnoses that fall under other strands of proficiency. For example, is it possible that it could be an error of conceptual understanding? Experience talking 
to students about their mistakes suggests the possibility that this was not an unintentional procedural slip, but rather a confusion about what procedures are permissible under what circumstances. Indeed, there is a situation in which it is quite permissible to lose the factor of 2, namely in solving the equation

$$
2 x^{2}+4 x+4=0 .
$$

For a student with a weak grasp of the difference between equations and expressions, the superficial procedural similarity between solving equations and transforming expressions is a snare. Both procedures involve doing something to expressions connected by equals signs. We should consider the possibility that the failure to appreciate the fundamental difference between the two situations is a failure of understanding, not a failure in manipulative skills.

As for strategic competence, there is an important aspect of this error which the partial credit mentality fails to weigh sufficiently: in addition to making the original slip, the student failed to correct it. Stepping back from the solution and contemplating it as a whole suggests an obvious strategy for checking the solution, namely differentiating the answer. Even without actually carrying out the differentiation, a student with a strategic frame of mind might well wonder where all the necessary $2 \mathrm{~s}$ and $4 \mathrm{~s}$ in the denominator will come from.

What of adaptive reasoning? This is defined in [KSF01] as "capacity for logical thought, reflection, explanation, and justification". Such a capacity, in particular an attention to the meaning of the equals sign and a tendency to reflect on the assertion being made each time you put one down on paper, might have led the student to notice that the first line in the solution effectively declares a number to be half of itself.

Finally, there could be an overall failure of productive disposition, which is defined as "habitual inclination to see mathematics as sensible, useful, and worthwhile, coupled with a belief in the value of diligence and in one's own efficacy." Experience suggests that erroneous solutions like the one described here are often the product of a lack of any purpose other than moving the symbols in the correct way. The bicyclist has a fundamental sense of purpose that corrects the wobble; this is often lacking in algebra students.

\section{Assessing the Strands of Proficiency}

Many algebra assessments concentrate on assessing procedural fluency. Indeed, the common view of algebra among students is that it is nothing but procedural fluency. The idea that there are ideas in algebra comes as a surprise to many. How would we go about assessing some of the other strands? One way is to ask more multi-step questions and word problems that involve all strands at once. But such questions rarely make their way onto standardized assessments. There is also a need for simple questions that assess the non-procedural aspects of algebraic proficiency. Here are a couple of suggested questions.

\section{Conceptual understanding}


Question. In the following problems, the solution to the equation depends on the constant $a$. Assuming $a$ is positive, what is the effect of increasing $a$ on the value of the solution? Does the solution increase, decrease, or remain unchanged? Give a reason for your answer that can be understood without solving the equation.

(a) $x-a=0$

(b) $a x=1$

(c) $a x=a$

(d) $\frac{x}{a}=1$

\section{Answer.}

(a) Increases. The larger $a$ is, the larger $x$ must be to give 0 when $a$ is subtracted from it.

(b) Decreases. The larger $a$ is, the smaller $x$ must be to give a product of 1 .

(c) Remains unchanged. As a changes, the two sides of the equation change together and remain equal.

(d) Increases. The larger $a$ is, the larger $x$ must be to give a ratio of 1 .

The equations in this problem are all trivially easy to solve. Nonetheless, students find this question difficult because they are not being asked to demonstrate a procedure, but rather to frame an explanation in terms of what it means for a number to be a solution to an equation. It is possible for students to learn the mechanics of solving equations without ever picking up the rather difficult concept of an equation as a statement of equality between two expressions whose truth is contingent upon the value of the variables, and the process of solving an equation as a series of logical inferences involving such statements. Asking students to reason about equations without solving them could assess this area of proficiency.

\section{Strategic competence}

Question. A street vendor of t-shirts finds that if the price of a t-shirt is set at $\$ p$, the profit from a week's sales is

$$
(p-6)(900-15 p) .
$$

Which form of this expression shows most clearly the maximum profit and the price that gives that maximum?
A. $(p-6)(900-15 p)$
B. $-15(p-33)^{2}+10935$
C. $-15(p-6)(p-60)$ 
D. $-15 p^{2}+990 p-5400$

Answer. B. Since $(p-33)^{2}$ is a square, it is always positive or zero, and it is only zero when $p=33$. In the expression for the profit, a negative multiple of this square is added to 10,935 . Thus the maximum profit is $\$ 10,935$, and the price which gives that profit is $\$ 33$.

In this problem the student is not asked to perform the manipulations that produce the different forms, but rather to show an understanding of why you might want to perform those manipulations, and which manipulations would be the best to choose for a given purpose. A similar question could be asked about form (C), which shows prices that produce zero profit, and form (A), which exhibits the profit as a product of two terms each of which has meaning in terms of the context of the original problem, allowing a student to infer that the production cost per item is $6 \$$, for example, or that the demand function is given by the expression $900-15 p$.

Such questions invite students to contemplate the form of an algebraic expression and to formulate ideas about the possible purposes to which that form is adapted. Too often, students feel they must instantly do something to an expression, without having formulated a purpose.

\section{Conclusion}

Algebra is at core about proficiency with symbolic representations. Assessment of this proficiency should include fluency with symbolic manipulations, but should also include the other four strands of mathematical competence. Such a richer assessment is often unfortunately delayed until functions have been introduced, since the different ways of representing functions, and their numerous application in real-world contexts, provide good ground for the formulation of more conceptual and strategic questions. Simple questions of this nature at the level of elementary algebra are hard to come by, with the result that it is often taught as a purely procedural skill, with its more conceptual and strategic aspects either ignored or veiled behind the more abstract concept of function.

\section{References}

[KSF01] Jeremy Kilpatrick, Jane Swafford, and Bradford Findell (eds.), Adding it up: Helping children learn mathematics, National Academy Press, Washington, DC, 2001.

[RAN03] RAND Mathematics Study Panel, Deborah Loewenberg Ball, Chair, Mathematical proficiency for all students: toward a strategic research and development program in mathematics education, RAND, Santa Monica, 2003. 